\title{
Identification of the potential roles of ring finger protein 8 in TP53-mutant breast cancer
}

\author{
FENG ZHAO ${ }^{1,2^{*}}$, PEIBIN WANG $^{1,2^{*}}$, YAN GUO $^{3 *}$, QI LU $^{1,2}, \mathrm{XU} \mathrm{KONG}^{1,2}$, \\ DONGWEI SU ${ }^{4}$, HENGYU LI ${ }^{4}$, GUOPING LIU ${ }^{4}$ and CHAOQIAN LIU ${ }^{4}$
${ }^{1}$ Anhui Province Engineering Laboratory of Occupational Health and Safety, Anhui University of Science and Technology, Huainan, Anhui 232000; ${ }^{2}$ Department of General Surgery, Huainan First People's Hospital Affiliated to Bengbu Medical College, Huainan, Anhui 232000; Departments of ${ }^{3}$ Endocrinology and ${ }^{4}$ General Surgery, Changhai Hospital, Second Military Medical University, Shanghai 200433, P.R. China

Received September 19, 2019; Accepted October 22, 2020

DOI: $10.3892 / \mathrm{ol} .2020 .12303$

\begin{abstract}
Breast cancer is one of the malignant tumors with the highest mortality rate. With the development of precise treatment technology for cancer, numerous molecular targets have been identified and applied in the treatment of diseases. The present study investigated the potential role of ring finger protein 8 (RNF8) in TP53-mutant breast cancer and explored its possible mechanisms of action through a combination of bioinformatics techniques and cell biology. The results revealed that significantly different genes were expressed in RNF8-knockout mice sequencing data compared with in the control group in the presence ofTP53 mutations. Downregulated genes were significantly enriched in several pathways of cell proliferation and apoptosis regulation, development and transcription regulation, while upregulated genes were mainly enriched in immune response-associated signaling pathways. Therefore, the consensus genes of the major signaling pathways were further analyzed, revealing that among patients with TP53 wild-type breast cancer, the prognosis of patients with low expression levels of fibroblast growth factor receptor 1, LIM homeobox 2 and EPH receptor B2 was improved compared with that of patients with high expression levels, while among patients with TP53-mutant breast cancer, there was no significant difference in survival status. In addition, among patients with TP53-mutant breast cancer, the prognosis of patients with high BR serine/threonine
\end{abstract}

Correspondence to: Dr Guoping Liu or Dr Chaoqian Liu, Department of General Surgery, Changhai Hospital, Second Military Medical University, 168 Changhai Road, Shanghai 200433, P.R. China

E-mail: lgp2680@sina.com

E-mail: liuchaoqian2002@aliyun.com

${ }^{*}$ Contributed equally

Key words: ring finger protein 8, TP53 mutant, breast cancer, LIM homeobox 2, EPH receptor B2, BR serine/threonine kinase 1 kinase 1 expression was significantly improved compared with that in patients with low expression. Finally, cell biology experiments demonstrated that in TP53-mutant breast cancer cells (HCC1937), inhibition of RNF8 significantly inhibited the proliferation of TP53-mutant HCC1937 cells and promoted their apoptosis. The present findings may enrich the understanding of the role of RNF8 and indicated that RNF8 may be used as a potential molecular target in TP53-mutant breast cancer, which may lead to the development of clinical treatment strategies.

\section{Introduction}

Breast cancer is one of the most common types of cancer in the world. Breast cancer cases in the world have continued to increase from 2005 , with $12 \%$ of women expected to develop this disease in their lifetime (1). Ongoing research on breast cancer is trying to improve its treatment to assist in dealing with the issue (2).

Ring finger protein 8 (RNF8) is a gene in the human body that encodes the E3 ubiquitin-protein ligase RNF8 enzyme (3). It is involved in various activities of the body associated with immune system functions and DNA repair $(4,5)$. Previous studies have indicated that RNF8 is a damage-responsive protein that integrates both protein phosphorylation and ubiquitylation, serving an essential role in disease treatment $(6,7)$. Additionally, depletion of RNF8 in the body can lead to cell cycle arrest and inhibition of cell proliferation (8).

The vital role of RNF8 in diseases is evident in the treatment of various types of cancer (9). It was reported that RNF8 can act as a co-activator to promote cell proliferation of estrogen receptor (ER) $\alpha$-positive breast cancer via post-transcriptional signaling pathways (8). Furthermore, RNF8 was reported to promote epithelial-mesenchymal transition of breast cancer cells and facilitate the metastasis of breast cancer cells in vivo (10). By contrast, another study demonstrated that mice with RNF8 deficiency have an increased risk of mammary tumorigenesis, since RNF8 can regulate Notch signaling by ubiquitylating the active NOTCH1 protein, leading to its degradation (7), while abnormal activation of Notch signaling would promote the risk of breast cancer. 
Additionally, Gao et al (11) revealed that RNF8 can negatively regulate the activation of NF- $\mathrm{\kappa B}$, of which excess activation can lead to cancer. Therefore, there are controversial roles of RNF8 in tumorigenesis, and the function of RNF8 in breast cancer remains unclear.

In the present study, a series of bioinformatic approaches were used to analyze breast cancer sequencing data to explore the underlying mechanism of RNF8 in breast cancer. Subsequently, further experiments were performed to verify the bioinformatic results. The present study suggested that breast cancer progression may be closely associated with RNF8 expression

\section{Materials and methods}

Bioinformatics analysis. RNA sequencing data from TP53-mutant mammary tumor cells was downloaded from the GSE76075 dataset containing mRNA sequencing data for two independent mouse TP53-mutant breast cancer cell lines (RNF8 ${ }^{-/}$and RNF8-wild-type) (12) from the Gene Expression Omnibus (GEO) database (https://www.ncbi.nlm. nih.gov/geo/query/acc.cgi?acc $=$ GSE76075). The gene names and gene count files were obtained, and the GFOLD values of each gene were calculated using the open source GFOLD software v1.1.4 (13). A total of 500 differentially expressed genes with the largest (upward) and minimum (downward) GFOLD values were selected, and Gene Ontology (GO) enrichment analysis was performed on these genes using the Database for Annotation, Visualization and Integrated Discovery (DAVID; https://david.ncifcrf.gov/). Furthermore, the GO enrichment results of each group were clustered using the GOSemSim v3.11 R package (http://www.bioconductor. org/packages/release/bioc/html/GOSemSim.html) (14), and the clustering results between the groups were visualized using the ggtree v3.11 R package (http://www.bioconductor. org/packages/release/bioc/html/ggtree.html) (15). Subsequently, survival analysis of key genes was performed using Kaplan-Meier (KM) Plotter (http://kmplot.com/analysis/) with the log-rank test.

Cell culture. The HCC1937 cell line was purchased from the China Infrastructure of Cell Line Resources (Institute of Basic Medical Sciences; Chinese Academy of Sciences). The HCC1937 cell line was selected due to its TP53-negative status, which can eliminate the uncertainty derived from TP53 mutations. Using the HCC1937 cell line helps to maintain consistency between human and mouse cell models. The cells were cultured in the RPMI 1640 (Thermo Fisher Scientific, Inc.) medium containing 10\% FBS (Gibco; Thermo Fisher Scientific, Inc.) at $37^{\circ} \mathrm{C}$ in the presence of $5 \% \mathrm{CO}_{2}$.

Cell transfection. According to the sequence of the RNF8 gene, three small interfering (si)RNAs (Table I) with different target sequences of RNF8 were synthesized by Shanghai Shenggong Biology Engineering Technology Service, Ltd., to decrease the expression levels of the RNF8 gene in the TP53-mutant HCC1937 cells. Untreated cells were used as negative control 1.A non-specific scrambled siRNA $(50 \mathrm{nM})$ was used as negative control 2 and specific siRNAs (siRNA1, siRNA2 and siRNA3; $50 \mathrm{nM}$ ), as well as a siRNA mix (consisting of all three specific siRNAs) against RNF8 were directly transfected into HCC1937 cells using
Table I. siRNA target sequences.

\begin{tabular}{ll}
\hline siRNAs & \multicolumn{1}{c}{ Sequence } \\
\hline siRNA1 & 5'-UGCGGAGUAUGAAUAUGAAUU-3' \\
siRNA2 & 5'-GGACAAUUAUGGACAACAAGA-3' \\
siRNA3 & 5'-UAAGGAGAAUGCGGAGUAT-3' \\
Control & 5'-GGUAUCGGCUUAUCAGUCCGAGUAATT-3'
\end{tabular}

siRNA, small interfering RNA.

Lipofectamine ${ }^{\circledR} 2000$ (Invitrogen; Thermo Fisher Scientific, Inc.) according to the manufacturer's protocol. Transfected cells were cultured at $37^{\circ} \mathrm{C}$ in the presence of $5 \% \mathrm{CO}_{2}$ for $48 \mathrm{~h}$, after which subsequent experiments were performed.

$R N A$ isolation, reverse transcription-quantitative ( $R T-q) P C R$ assay and western blot analysis. Total RNA from cells was extracted using the MiniBEST Universal RNA Extraction kit (Takara Bio,Inc.), and RT was performed using the PrimeScript RT reagent kit (Takara Bio, Inc.) according to the manufacturer's protocol. The resulting cDNA was stored at $-80^{\circ} \mathrm{C}$ until further use. For qPCR, the TB Green Fast qPCR mix (Takara Bio, Inc.) was used to quantify the expression levels of the corresponding genes on the ABI 7500 (Applied Biosystems; Thermo Fisher Scientific, Inc.), and the primer sequences of the detected genes are shown in Table II. The thermocycling conditions comprised an initial denaturation step at $95^{\circ} \mathrm{C}$ for $30 \mathrm{sec}$, followed by 40 cycles of amplification at $95^{\circ} \mathrm{C}$ for $5 \mathrm{sec}$ and $60^{\circ} \mathrm{C}$ for $30 \mathrm{sec}$. The relative transcript levels were quantified using the $2^{-\Delta \Delta \mathrm{Cq}}$ method (16) with GAPDH used as an internal control.

For the western blot experiments, cells were harvested and collected via centrifugation at $800 \mathrm{x}$ g for $3 \mathrm{~min}$ at room temperature. Cell proteins were lysed in NP-40 Lysis-Buffer [150 mM NaCl, 1\% NP-40 and $50 \mathrm{mM}$ Tris (pH 8.0)] and analyzed via BCA protein assay (Beyotime Institute of Biotechnology). Proteins were denatured by heating for $5 \mathrm{~min}$ at $85^{\circ} \mathrm{C}$, separated via $12 \%$ SDS-PAGE (50 $\mu$ g protein/lane) and transferred to PVDF membranes. Subsequently, the membranes were blocked with 5\% BSA (Beyotime Institute of Biotechnology) at room temperature for $30 \mathrm{~min}$ and incubated with anti-RNF8 (1:1,000; EMD Millipore; cat. no. 09-813), anti-GAPDH (1:5,000; BIOSS; cat. no. bsm-33033M), anti-EPH receptor B2 (EPHB2; 1:1,000; Sino Biological, Inc.; cat. no. 100091-T08) anti-LIM homeobox 2 (LHX2; 1:1,000; BIOSS; cat. no. bs-11200R;) and anti-BR serine/threonine kinase 1 (BRSK1; 1:1,000; BIOSS; cat. no. bs-7905R;) primary antibodies at $4^{\circ} \mathrm{C}$ overnight. After washing three times with PBS-Tween (0.1\% Tween-20), membranes were incubated with HRP-conjugated goat anti-rabbit IgG (1:10,000; BIOSS; cat. no. bs-0295G-HRP) and goat anti-mouse IgG secondary antibodies (1:10,000; BIOSS; cat. no. bs-0368G-HRP) at room temperature for $2 \mathrm{~h}$. ECL luminous solution (Bio-Rad Laboratories, Inc.) was used to detect the bands.

Cell proliferation assay. Cell Counting Kit-8 (CCK-8; Takara Bio, Inc.) was used to evaluate cell proliferation. Briefly, 
Table II. Primer sequences of the target genes.

\begin{tabular}{lll}
\hline Gene name & \multicolumn{1}{c}{ Forward primer } & \multicolumn{1}{c}{ Reverse primer } \\
\hline RNF8 & 5'-AAGCGACGGCAGCAGAA-3' & 5'-AGCACCTTCACCTTCCTCAG-3' \\
GAPDH & 5'-GCACCGTCAAGGCTGAGAAC-3' & 5'-TGGTGAAGACGCCAGTGGA-3' \\
EPHB2 & 5'-TGAGTGCCCTCAGATGGTCAA-3' & 5'-AGGGCAGGGTATCACAGTGAATG-3' \\
LHX2 & 5'-GGAAGCATCTACTGCAAGGAAG-3' & 5'-GAGGTGATAAACCAAGTCCCG-3' \\
BRSK1 & 5'-CGGGAACTTCATCTCCTTGGAC-3' & 5'-ACAGCACACTGTGACTCAGGCT-3'
\end{tabular}

RNF8, ring finger protein 8; LHX2, LIM homeobox 2; EPHB2, EPH receptor B2; BRSK1, BR serine/threonine kinase 1.

$5 \times 10^{2}$ cells/well were seeded into 96 -well plates and cultured in medium containing $10 \%$ FBS for $24,48,72$ or $96 \mathrm{~h}$ at $37^{\circ} \mathrm{C}$ in the presence of $5 \% \mathrm{CO}_{2}$. After $2 \mathrm{~h}$ of culture with the CCK-8 reagent, the cells were analyzed at a wavelength of $450 \mathrm{~nm}$ using a microplate reader.

Flow cytometric assay. The pretreated cells were harvested by trypsinization and fixed with $70 \%$ ethanol at $4{ }^{\circ} \mathrm{C}$ for $2 \mathrm{~h}$. Subsequently, the cells were stained with $10 \mu \mathrm{l}$ Annexin V-FITC and $5 \mu \mathrm{l}$ propidium iodide (both Shanghai Yeasen Biotechnology Co., Ltd.) for $1 \mathrm{~h}$ at room temperature. Finally, cells were analyzed using a FACS instrument (FACSCelesta; BD Biosciences) and corresponding data were analyzed using the FlowJo v10.6.2 software (FlowJo LLC).

Statistical analysis. All experiments were performed in triplicate. GraphPad Prism 8.0 (GraphPad Software Inc.) was used for statistical analysis. The data are presented as the mean \pm SD. The difference between two groups was analyzed by unpaired Student's t-test, while the comparisons among $>2$ groups were performed by one-way ANOVA followed by Bonferroni's multiple comparisons test as the post hoc test. $\mathrm{P}<0.05$ was considered to indicate a statistically significant difference.

\section{Results}

Transcriptome analysis of $\mathrm{RNF}^{-/}$TP53-mutant cells. To evaluate the signaling pathways affected by RNF8-knockdown in TP53-mutant breast cancer cells, the GSE76075 dataset was downloaded from the GEO database, containing mRNA sequencing data for two independent mouse TP53-mutant breast cancer cell lines ( $\mathrm{RNF}^{-/}$and RNF8-wild-type). The reads per kilobase of exon per million mapped sequence reads (RPKM) values were used to quantify the expression intensity of the genes, and the GFOLD values were used to evaluate the degree of differential gene expression, and a heat map was used to present the differential expression between the two cell lines. The results revealed that the GFOLD values of most of the genes between the two samples had different expression patterns (Fig. 1A). In addition, GFOLD values $>1$ or $<-1$ were used as the threshold for differential expression. The results revealed that there were 2,932 upregulated genes $(\sim 12 \%)$ and 2,255 downregulated genes $(\sim 10 \%)$ in $\mathrm{RNF}^{-/}$cells compared with in wild-type cells (Fig. 1B and C).
Enrichment and clustering. To further analyze the molecular mechanism of RNF8 in TP53-mutant breast cancer, a GO enrichment analysis of the top 500 downregulated and upregulated genes among the differentially expressed genes was performed using DAVID. The GO analysis yielded a large number of terms, making it difficult to quickly extract effective information; therefore, statistically significant GO terms were clustered by semantic similarity analysis. The results revealed that in RNF8-knockout TP53-mutant breast cancer cells, downregulated genes were significantly enriched in several pathways involved in cell proliferation and apoptosis regulation, development and transcription regulation, while upregulated genes were mainly enriched in immune response-associated pathways (Fig. 2).

Survival analysis based on key genes. A previous study has demonstrated that RNF8 is involved in the reproductive development of the body (17). Therefore, the present study investigated whether RNF8 regulated genes in development-associated pathways in TP53-knockout breast cancer cells. A statistical analysis of all the genes enriched in the GO 'development' cluster was performed, and genes that appeared in $>3$ GO terms were selected. Finally, a total of 10 genes were obtained, namely Myosin Heavy Chain 10, Paired Box 6, LHX2, GATA Binding Protein 3, Fibroblast Growth Factor Receptor 1 (FGFR1), EPHB2, Sphingosine-1-Phosphate Receptor 1, Empty Spiracles Homeobox 2, BRSK1 and GDNF family receptor $\alpha-2$. Subsequently, the association between these 10 genes and the prognosis in patients with breast cancer was analyzed using KM Plotter (Table III). The results revealed that in patients with TP53-wild-type breast cancer, the prognosis in patients with low expression levels of LHX2 and EPHB2 was significantly improved compared with that in patients with high expression ( $\mathrm{LHX} 2, \mathrm{P}=0.00045$; $\mathrm{EPHB} 2$, $\mathrm{P}=0.024$; Fig. $3 \mathrm{~A}$ and $\mathrm{B}$ ), while the prognosis in patients with low expression levels of FGFR1 was significantly decreased compared with that in patients with high expression (FGFR1, $\mathrm{P}=0.026$; Fig. 3D). In patients with TP53-mutant breast cancer, there was no significant difference in survival status, except for the prognosis in patients with high BRSK1 expression, which was significantly improved compared with that in patients with low expression ( $\mathrm{P}=0.0014$; Fig. 3C).

RNF8-knockdown inhibits cell proliferation and promotes apoptosis in human TP53-mutant breast cancer cells. To further elucidate the role of RNF8 in TP53-mutant breast cancer, 


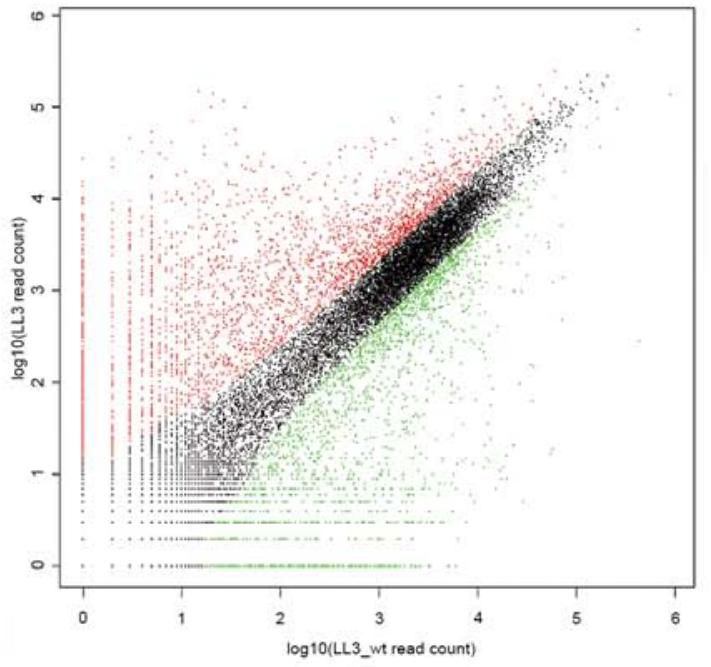

B

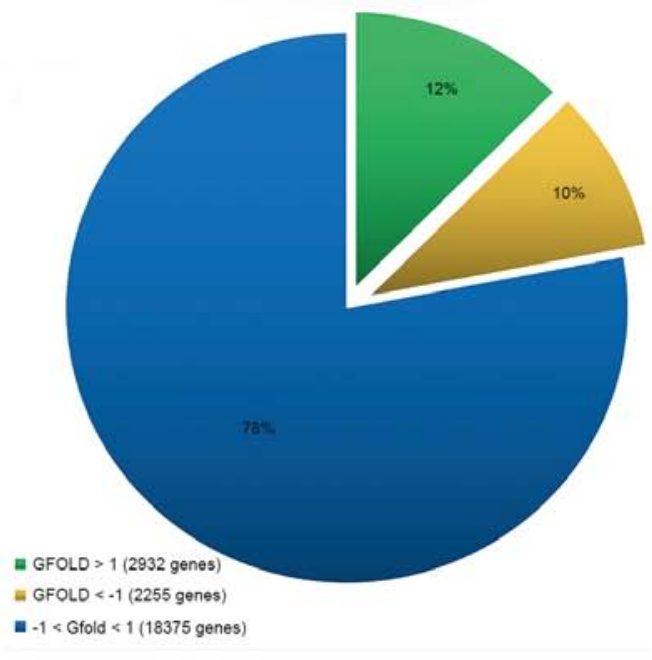

C

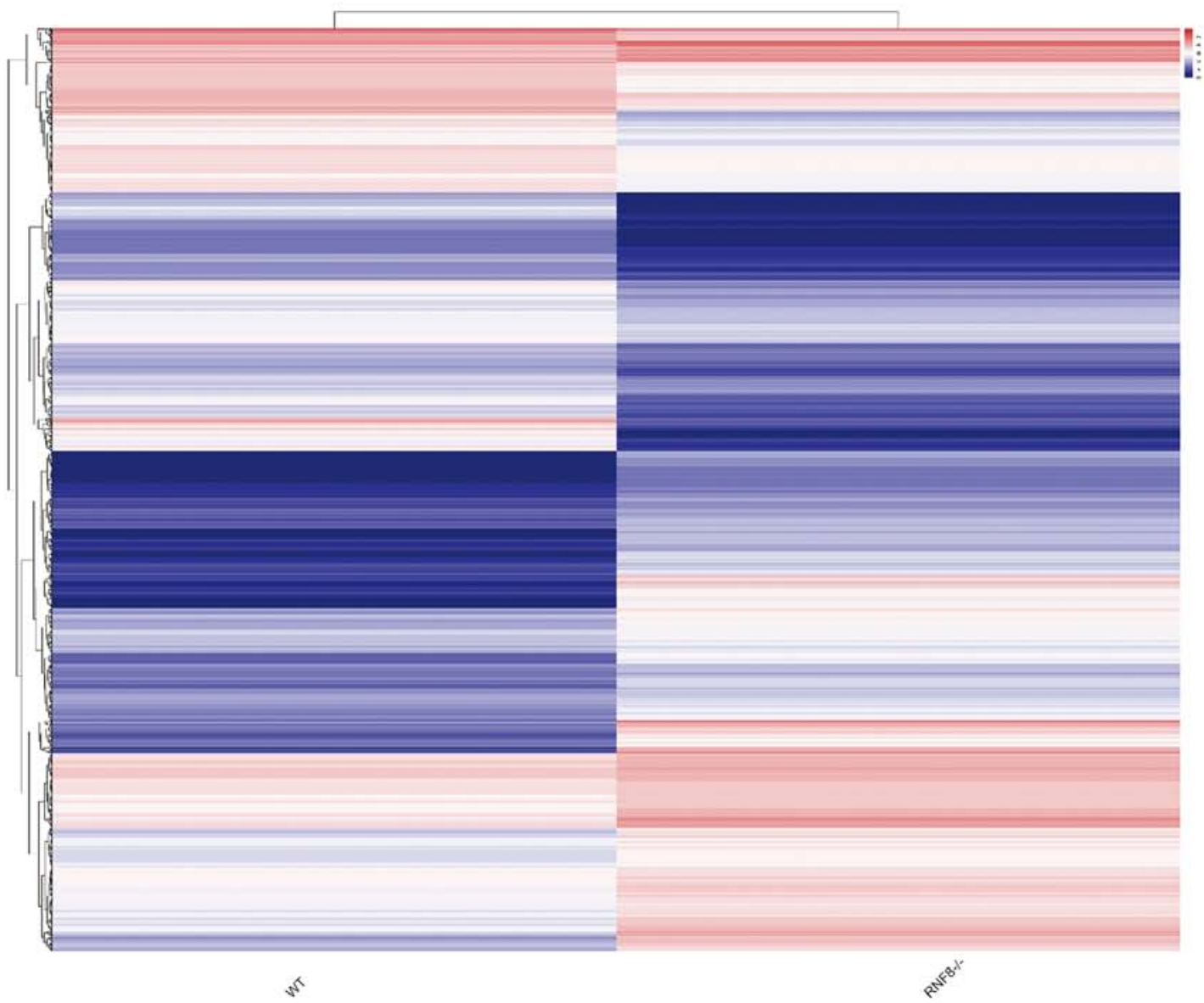

Figure 1. (A) Differentially expressed genes in the GSE76075 dataset. This dataset defines the RNF8-knockdown and p53-mutant mouse breast cancer cell line as LL3. The X-axis represents the $\log 10$ (WT read count), while the y-axis represents the log10 (Mutation read count), and each dot represents a gene. Red dots represent upregulated genes, while green dots represent downregulated genes and black dots represent non-differentially expressed genes. (B) Distribution of GFOLD values of differentially expressed genes. Blue represents the proportion of genes with a GFOLD value in the range of -1 to 1 , green represents the proportion of genes with a GFOLD value $>1$ and yellow represents the proportion of genes with a GFOLD value $<-1$. (C) Heat map of the expression of the top 500 genes with the largest or smallest GFOLD values in WT and mutant RNF8 samples. WT, wild-type; RNF8, ring finger protein 8.

RNF8 mRNA and protein expression was knocked down in the TP53-mutant human breast cancer HCC1937 cell line using three different siRNAs and a siRNA mix consisting of a; three siRNAs (Fig. 4A) In order to achieve the best interfering 
Table III. Association between 10 differentially expressed genes and the prognosis in patients with breast cancer.

\begin{tabular}{lcccrcc}
\hline Gene & Count & Frequency, $\%$ & GFOLD & Rank & $\begin{array}{c}\text { Kaplan-Meier analysis } \\
\text { P-value (TP53-mutated) }\end{array}$ & $\begin{array}{c}\text { Kaplan-Meier analysis } \\
\text { P-value (TP53-wild-type) }\end{array}$ \\
\hline MYH10 & 5 & 45.45 & -6.07418 & 196 & 0.57000 & 0.77000 \\
PAX6 & 4 & 36.36 & -7.30754 & 98 & 0.17000 & 0.84000 \\
LHX2 & 4 & 36.36 & -7.12856 & 116 & 0.60000 & 0.00045 \\
GATA3 & 4 & 36.36 & -4.67309 & 372 & 0.37000 & 0.62000 \\
FGFR1 & 4 & 36.36 & -4.43547 & 418 & 0.81000 & 0.05800 \\
EPHB2 & 3 & 27.27 & -7.27086 & 101 & 0.49000 & 0.02400 \\
S1PR1 & 3 & 27.27 & -7.51289 & 82 & 0.52000 & 0.74000 \\
EMX2 & 3 & 27.27 & -5.30267 & 286 & 0.86000 & 0.51000 \\
BRSK1 & 3 & 27.27 & -6.58582 & 153 & 0.00140 & 0.23000 \\
GDNF & 3 & 27.27 & -5.50851 & 254 & 0.61000 & 0.66000 \\
\hline
\end{tabular}

MYH10, Myosin Heavy Chain 10; PAX6, Paired Box 6; LHX2, LIM Homeobox 2; GATA3, GATA Binding Protein 3; FGFR1, Fibroblast Growth Factor Receptor 1; EPHB2, EPH Receptor B2; S1PR1, Sphingosine-1-Phosphate Receptor 1; EMX2, Empty Spiracles Homeobox 2; BRSK1, BR serine/threonine kinase 1; GDNF, GDNF family receptor $\alpha-2$.
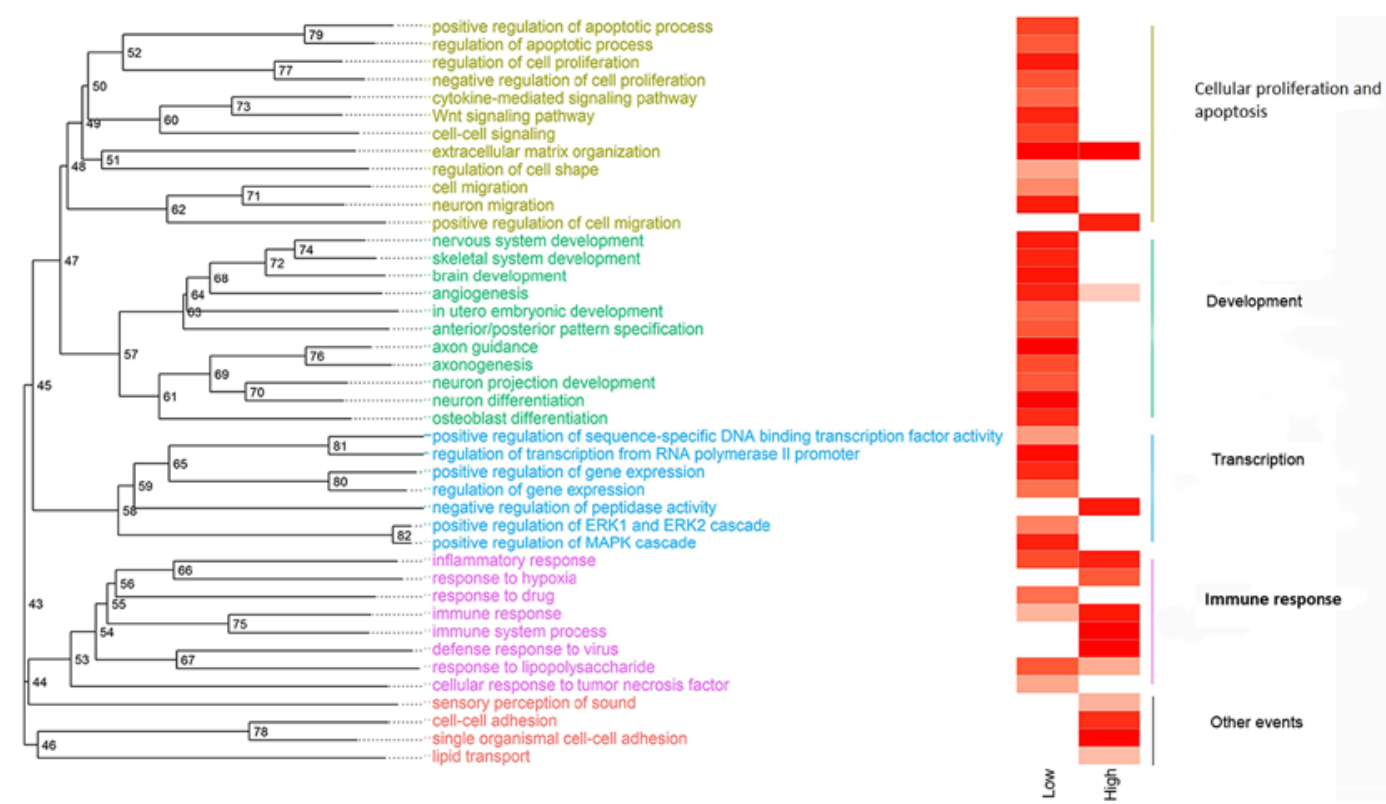

Figure 2. Gene Ontology term clusters of upregulated and downregulated genes in ring finger protein 8-knockdown/TP53-mutant breast cancer cells. Enrichment P-values are represented by the red scale.

effect, the siRNA mix was used in subsequent experiments. The results demonstrated that RNF8-knockdown in HCC1937 cells significantly decreased cell proliferation compared with that in control cells (Fig. 4B). Since the aforementioned bioinformatics analysis revealed that downregulated genes after RNF8-knockout were mainly enriched in apoptosis-associated signaling pathways, the effect of RNF8-knockdown in siRNA-treated HCC1937 cells was compared with that in control siRNA-treated HCC1937 cells using flow cytometry. The results demonstrated that apoptosis in HCC1937 cells with RNF8-knockdown was significantly higher compared with that in control cells (Fig. 4C). Overall, the present results indicated that downregulation of RNF8 expression inhibited proliferation and enhanced apoptosis in TP53-mutant breast cancer cells.
qPCR and western blot analysis of the expression levels of LHX2, EPHB2 and BRSK1 in TP53-mutant human breast cancer cells with low RNF8 expression. Since low FGFR1 expression was associated with a poor prognosis, which indicated that it may have a different function compared with LHX2 and EPHB2 in breast cancer, FGFR1 was excluded from subsequent evaluations. The association between RNF8 and the three aforementioned genes associated with prognosis, LHX2, EPHB2 and BRSK1, was based on sequencing data from mouse breast cancer cells. To verify whether knockdown of RNF8 in human breast cancer cells also caused a decrease in the expression levels of LHX2, EPHB2 and BRSK1, qPCR was performed, revealing that the expression levels of EPHB2, BRSK1 and LHX2 were significantly downregulated in human 

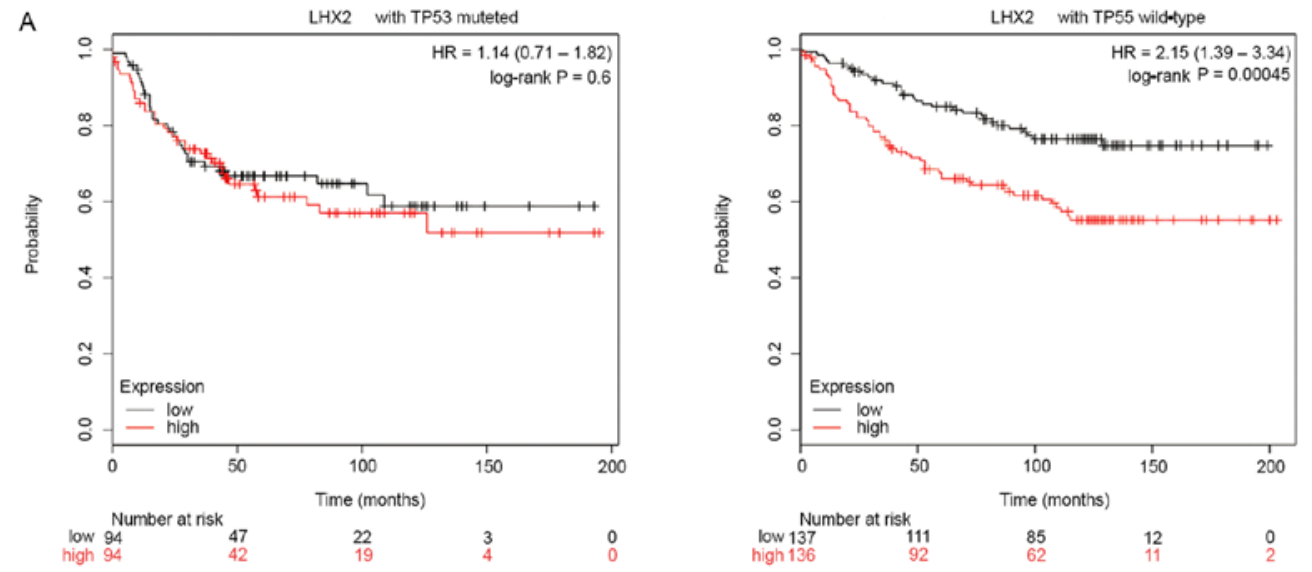

B
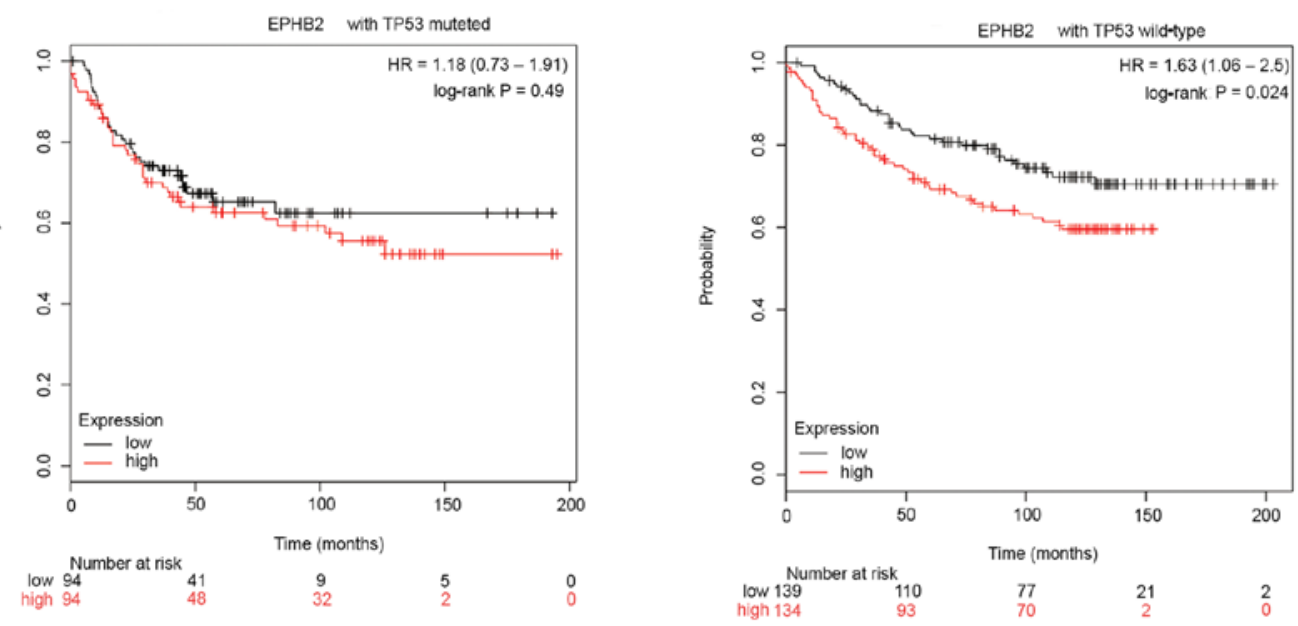

$\mathrm{C}$
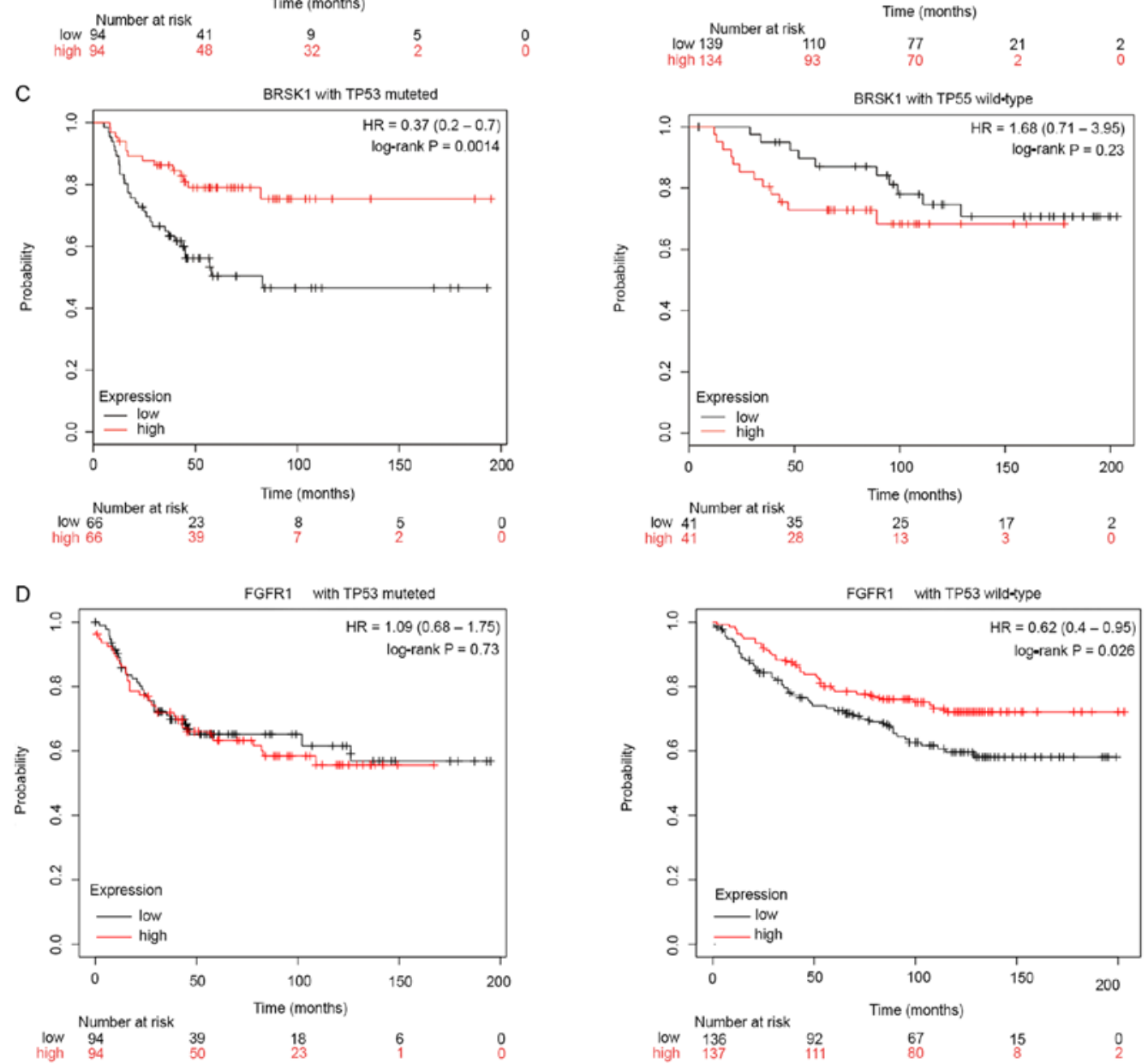

Figure 3. Kaplan-Meier curve analysis of overall survival based on Kaplan-Meier plotter mRNA expression levels of (A) LHX2, (B) EPHB2, (C) BRSK1 and (D) FGFR1 in patients with breast cancer. HR, hazard ratio; LHX2, LIM homeobox 2; EPHB2, EPH receptor B2; BRSK1, BR serine/threonine kinase 1; FGFR1, fibroblast growth factor receptor 1. 

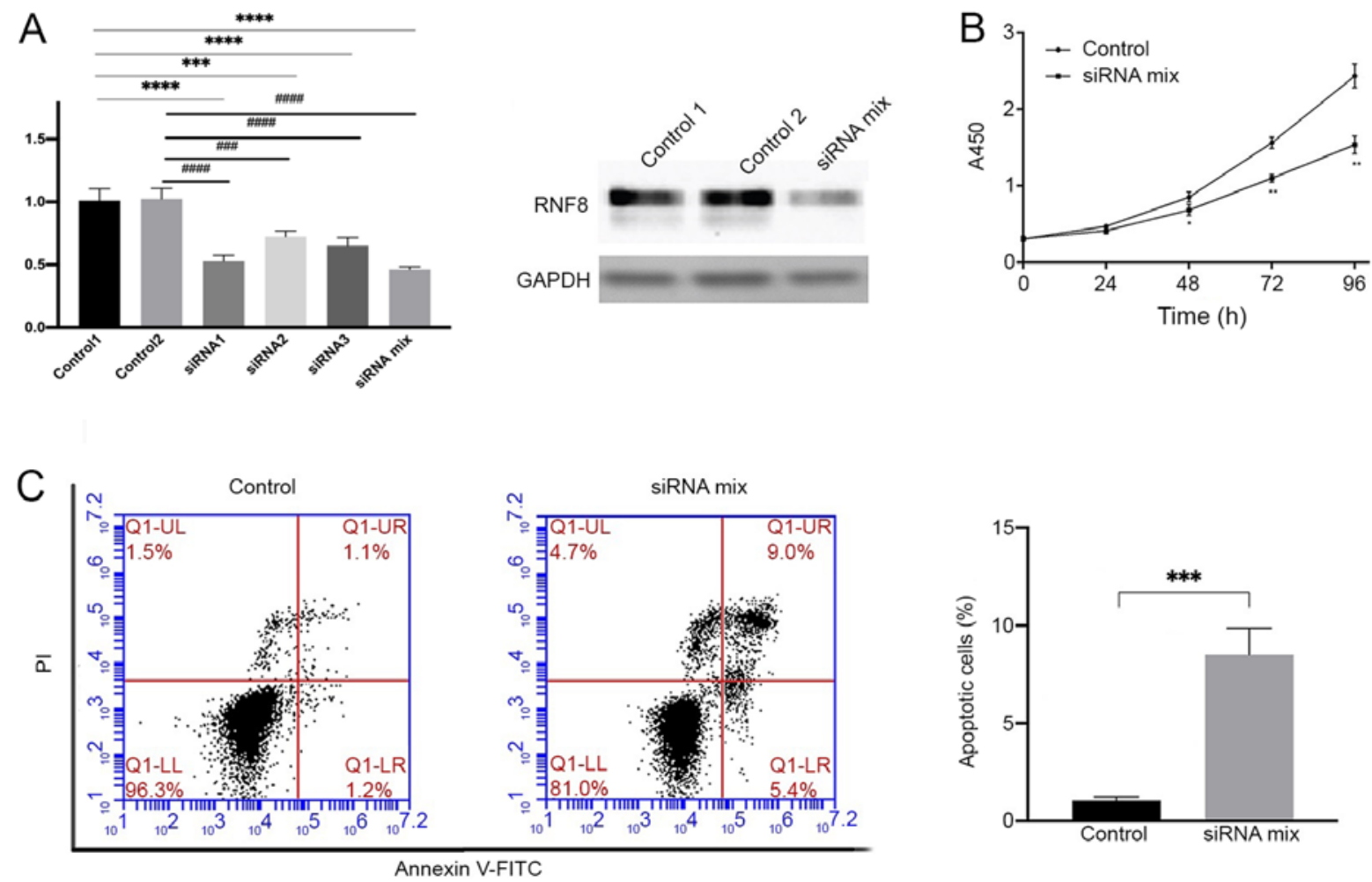

Figure 4. (A) RNF8 mRNA and protein expression using siRNAs. siRNA1, siRNA2 and siRNA3 are siRNAs targeting different RNF8 sequences, while siRNA mix is a mixture of siRNA1, siRNA2 and siRNA3. Control 1 is the untreated group and control 2 is the non-sense siRNA treatment group. ${ }^{* * *} \mathrm{P}<0.001$ and ${ }^{* * * * *} \mathrm{P}<0.0001$ vs. Control 1. ${ }^{\# \# \#} \mathrm{P}<0.001$ and ${ }^{\# \# \# \#} \mathrm{P}<0.0001$ vs. Control 2. Significance was assessed using one-way ANOVA with Bonferroni's multiple comparisons post-hoc test. (B) Cell proliferation curves. Control represents the non-sense siRNA treatment group. ${ }^{*} \mathrm{P}<0.05$ and ${ }^{* * *} \mathrm{P}<0.01$ vs. control. Significance was assessed using an unpaired Student's t-test. (C) Annexin V-FITC/PI staining was used to analyze cell apoptosis. ${ }^{* * *} \mathrm{P}<0.001$. Significance was assessed using an unpaired Student's t-test. Results are presented as the mean \pm SD $(n=3)$. siRNA, small interfering RNA; RNF8, ring finger protein 8; A, absorbance.

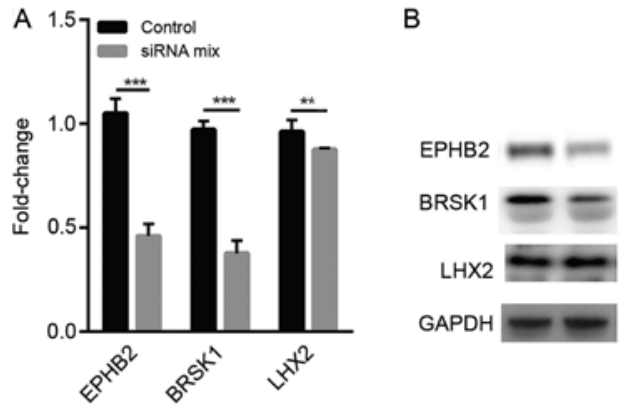

Figure 5. (A) mRNA and (B) protein expression levels of LHX2, EPHB2 and BRSK1 in siRNA-ring finger protein 8 and control siRNA groups. Results are presented as the mean $\pm S D(n=3) .{ }^{* *} \mathrm{P}<0.01 ;{ }^{* * * *} \mathrm{P}<0.001$. Significance was assessed using an unpaired Student's t-test. siRNA, small interfering RNA; LHX2, LIM homeobox 2; EPHB2, EPH receptor B2; BRSK1, BR serine/threonine kinase 1.

breast cancer HCC1937 cells after treatment with the siRNA mix (Fig. 5A). A similar trend was also observed in protein expression in RNF8-silenced cells (Fig. 5B).

\section{Discussion}

Previous studies have indicated that TP53 mutations have important functions in multiple subtypes of breast cancer, such as triple-negative and HER2 ${ }^{+}$breast cancer (18-20). Similarly, some studies have reported that RNF8 is associated with the oncogenesis of breast cancer $(10,11)$. For example, Kuang et al (10) demonstrated that RNF8 promotes epithelial-mesenchymal transition in breast cancer cells (including TP53 wild-type and mutant cells); however, the aforementioned study did not investigate the mechanism of RNF8 promoting epithelial-mesenchymal transition in TP53-mutant breast cancer. Other studies have tried to investigate the specific mechanism of RNF8 in breast cancer $(21,22)$. For example, it has been reported that RNF8 cooperates with RNF168 to mediate Forkhead Box M1 ubiquitination and degradation in breast cancer (21); however, the aforementioned study used the MCF7 cell line, which is a TP53-wild-type breast cancer cell line. In the present study, different datasets were used to investigate the role of RNF8 in TP53-mutant breast cancer and to explore the specific mechanism of RNF8 promoting TP53-mutant breast cancer through transcriptome sequencing, and verified the co-expression of RNF8, LHX2 and EPHB2 in the HCC1937 cell line.

RNF8 is an E3 ubiquitin ligase that acts primarily on the DNA damage repair process and can rapidly accumulate at the site of DNA damage through Fork Head Associated domain-mediated phosphorylation of Mediator Of DNA Damage Checkpoint 1 (22-24). Numerous proteins associated with DNA repair, such as P53 binding protein and BRCA1, can accumulate and function through its signal amplification (25). Therefore, RNF8 is a key factor that serves an important role in telomere protection, maintaining genomic integrity and regulating the cell cycle (26). 
A previous study has revealed that the function of RNF8 is highly dependent on the P53 protein, suggesting that RNF8 serves a biological role in synergy with P53 (27). However, numerous studies have revealed a number of mutations in the P53 gene in tumor cells $(28,29)$; therefore, it is increasingly important to investigate the functional role of RNF8 under the condition of P53 mutation (27). The present study identified the differentially expressed genes in mouse transcriptome data from the GEO database by comparing the low and normal expression levels of RNF8 in the TP53-mutant samples, revealing that downregulated genes were mainly enriched in several pathways involved in cell proliferation and apoptosis regulation, development and transcription regulation. This is consistent with previous studies $(28,29)$, indicating that even in the presence of P53 mutations, the main function of RNF8 is still focused on the regulation of the cell cycle and transcription factors, thus confirming that the function of RNF8 is not completely P53-dependent and that TP53 mutations have a relatively small effect on the physiological function of RNF8.

The role of RNF8 in tumor development and progression exhibits a double-sided action, resulting in either tumor inhibition or promotion. In a mouse model, the downregulation or loss of RNF8 results in a significant increase of tumor incidence, including the incidence of leukemia in tumorigenesis of lymphoma (50\%), thymoma $(38 \%)$, mammary carcinoma (13\%), skin tumor $(13 \%)$ and sarcoma (13\%) (30). A previous study has revealed that the expression levels of P53 are significantly increased in RNF8-knockout mice (12). At the same time, RNF8 has a strong inhibitory effect on breast cancer and can downregulate the Notch signaling pathway by deleting the C-terminal of the NOTCH1 intracellular domain (7), thereby limiting the spread of mammary luminal progenitors. Data from The Cancer Genome Atlas also support a negative association between RNF8 expression and the Notch signaling pathway (7).

However, a previous study has revealed that decreased RNF8 expression can significantly improve radiotherapy sensitivity in nasopharyngeal cancer, while inhibiting cell proliferation and increasing apoptosis (31). In breast cancer MCF-7 cells, overexpression of RNF8 can significantly increase epithelial-mesenchymal transition and it mainly promotes phosphorylation of GSK $3 \beta$, further inhibiting its activity (10). In addition, an increase in phosphorylation of $\beta$-catenin has been observed in RNF8-overexpressing cells (10). Furthermore, in the treatment of breast cancer using tamoxifen to block ER $\alpha$, tamoxifen exerts anticancer effects mainly by blocking the ER $\alpha$ signaling pathway (31), but a previous study has revealed that RNF8 can activate the ER $\alpha$ signaling pathway and thus promote tumor cell proliferation (8). The aforementioned studies did not focus on p53 mutations. Therefore, the present study investigated the effects of RNF8 in TP53-mutant breast cancer cells, revealing that downregulation of RNF8 expression in a cell model with TP53 mutation significantly inhibited the proliferation of tumor cells. In addition, the current study hypothesized that the tumorigenesis and development of breast cancer may be regulated by a sophisticated network, and the role of RNF8 in the tumorigenesis of TP53-mutant breast cancer may not be unique. Therefore, future studies should further explore RNF8/TP53 and immune response-associated pathways (32).

\section{Acknowledgements}

Not applicable.

\section{Funding}

The present study was supported by the Natural Science Research Project Foundation in Higher Education of Anhui Province in China (grant nos. KJ2019A0094 and KJ2019A0095), the 5th '50 Science \& Technology Stars' Innovation Research Team of Huainan, Anhui Province in China [Huainan Talents (2018) 7], the Key Project of Natural Science Foundation of Bengbu Medical College in China (2019; grant no. BYKY2019318ZD) and the Science and Technology Program of Huainan, Anhui Province in China (grant no. 2018B59).

\section{Availability of data and materials}

The datasets used and/or analyzed during the current study are available from the corresponding author on reasonable request, and the GSE76075 dataset is available from the Gene Expression Omnibus database (https://www.ncbi.nlm.nih. gov/geo/query/acc.cgi?acc=GSE76075).

\section{Authors' contributions}

FZ performed the cell experiments, prepared the figures and wrote the manuscript. PW and YG performed the qPCR and western blot analysis, and data interpretation. QL and XK performed flow cytometry. DS and HL performed the bioinformatics analysis. GL and CL designed the study and revised the manuscript. All authors read and approved the final manuscript.

\section{Ethics approval and consent to participate}

Not applicable.

\section{Patient consent for publication}

Not applicable.

\section{Competing interests}

The authors declare that they have no competing interests.

\section{References}

1. Pilevarzadeh $\mathrm{M}$, Amirshahi $\mathrm{M}$, Afsargharehbagh $\mathrm{R}$, Rafiemanesh H, Hashemi SM and Balouchi A: Global prevalence of depression among breast cancer patients: A systematic review and meta-analysis. Breast Cancer Res Treat 176: 519-533, 2019.

2. Greten FR and Grivennikov SI: Inflammation and cancer: Triggers, mechanisms, and consequences. Immunity 51: 27-41, 2019.

3. Pederiva C and Farnebo M: RNF8-The Achilles heel of DNA repair when splicing rules. Cell Cycle 17: 137-145, 2018.

4. Jacobs JJ: Fusing telomeres with RNF8. Nucleus 3: 143-149, 2012. 
5. Stewart GS: Solving the RIDDLE of 53BP1 recruitment to sites of damage. Cell Cycle 8: 1532-1538, 2009.

6. Li F, Liu B, Zhou X and Xu Q: Silencing of E3 ubiquitin ligase RNF8 enhances ionizing radiation sensitivity of medulloblastoma cells by promoting the deubiquitination of PCNA. Oncol Res 26: 1365-1373, 2018

7. Li L, Guturi KKN, Gautreau B, Patel PS, Saad A, Morii M, Mateo F, Palomero L, Barbour H, Gomez A, et al: Ubiquitin ligase RNF8 suppresses Notch signaling to regulate mammary development and tumorigenesis. J Clin Invest 128: 4525-4542, 2018.

8. Wang S, Luo H, Wang C, Sun H, Sun G, Sun N, Zeng K, Song H, Zou R, Zhou T, et al: RNF8 identified as a Co-activator of estrogen receptor $\alpha$ promotes cell growth in breast cancer. Biochim Biophys Acta Mol Basis Dis 1863: 1615-1628, 2017.

9. Yamamoto T, Taira Nihira N, Yogosawa S, Aoki K, Takeda H, Sawasaki $\mathrm{T}$ and Yoshida K: Interaction between RNF8 and DYRK2 is required for the recruitment of DNA repair molecules to DNA double-strand breaks. FEBS Lett 591: 842-853, 2017.

10. Kuang J, Li L, Guo L, Su Y, Wang Y, Xu Y, Wang X, Meng S, Lei L, Xu L and Shao G: RNF8 promotes epithelial-mesenchymal transition of breast cancer cells. J Exp Clin Cancer Res 35: 88, 2016.

11. Gao $\mathrm{S}, \mathrm{Wu} \mathrm{J}$, Liang $\mathrm{L}$ and Xu R: RNF8 negatively regulates NF-kappaB signaling by targeting IkappaB kinase: Implications for the regulation of inflammation signaling. Biochem Biophys Res Commun 488: 189-195, 2017.

12. Xu G, Chapman JR, Brandsma I, Yuan J, Mistrik M, Bouwman P, Bartkova J, Gogola E, Warmerdam D, Barazas M, et al: REV7 counteracts DNA double-strand break resection and affects PARP inhibition. Nature 521: 541-544, 2015.

13. Feng J, Meyer CA, Wang Q, Liu JS, Shirley Liu X and Zhang Y: GFOLD: A generalized fold change for ranking differentially expressed genes from RNA-seq data. Bioinformatics 28 : 2782-2788, 2012.

14. Yu G: Gene Ontology Semantic Similarity Analysis Using GOSemSim. In: Kidder B. (ed). Stem Cell Transcriptional Networks. Methods Mol Biol 2117: 207-215, 2020.

15. Yu G, Lam TT, Zhu H and Guan Y: Two methods for mapping and visualizing associated data on phylogeny using ggtree. Mol Biol Evol 35: 3041-3043, 2018

16. Livak KJ and Schmittgen TD: Analysis of relative gene expression data using real-time quantitative PCR and the 2(-Delta Delta C(T)) method. Methods 25: 402-408, 2001

17. Li L, Halaby MJ, Hakem A, Cardoso R, El Ghamrasni S, Harding S, Chan N, Bristow R, Sanchez O, Durocher D and Hakem R: Rnf8 deficiency impairs class switch recombination, spermatogenesis, and genomic integrity and predisposes for cancer. J Exp Med 207: 983-997, 2010.

18. Giordano A, Liu Y, Armeson K, Park Y, Ridinger M, Erlander M, Reuben J, Britten C, Kappler C, Yeh E and Ethier S: Polo-like kinase 1 (Plk1) inhibition synergizes with taxanes in triple negative breast cancer. PLoS One 14: e0224420, 2019.

19. Petry V, Bonadio RC, Cagnacci AQC, Senna LAL, Campos RDNG, Cotti GC, Hoff PM, Fragoso MCBV and Estevez-Diz MDP: Radiotherapy-induced malignancies in breast cancer patients with TP53 pathogenic germline variants (Li-Fraumeni syndrome). Fam Cancer 19: 47-53, 2020.
20. Arturo JF, Chobrutskiy BI, Yeagley M, Patel DN, Falasiri S, Patel JS and Blanck G: Electrostatic complementarity of B-cell receptor CDR3s and TP53-mutant amino acids in breast cancer is associated with increased disease-free survival rates. Cell Mol Immunol 17: 776-778, 2020.

21. Kongsema M, Zona S, Karunarathna U, Cabrera E, Man EP, Yao S, Shibakawa A, Khoo US, Medema RH, Freire R and Lam EW: RNF168 cooperates with RNF8 to mediate FOXM1 ubiquitination and degradation in breast cancer epirubicin treatment. Oncogenesis 5: e252, 2016.

22. Lee HJ, Li CF, Ruan D, Powers S, Thompson PA, Frohman MA and Chan CH: The DNA damage transducer RNF8 facilitates cancer chemoresistance and progression through twist activation. Mol Cell 63: 1021-1033, 2016

23. Mandemaker IK, van Cuijk L, Janssens RC, Lans H, Bezstarosti K, Hoeijmakers JH, Demmers JA, Vermeulen W and Marteijn JA: DNA damage-induced histone $\mathrm{H} 1$ ubiquitylation is mediated by HUWE1 and stimulates the RNF8-RNF168 pathway. Sci Rep 7: 15353, 2017

24. Paul A and Wang B: RNF8-and Ube2S-dependent ubiquitin lysine 11-linkage modification in response to DNA damage. Mol Cell 66: 458-472.e5, 2017.

25. Yoshida K and Miki Y: Role of BRCA1 and BRCA2 as regulators of DNA repair, transcription, and cell cycle in response to DNA damage. Cancer Sci 95: 866-871, 2004.

26. Rai R, Li JM, Zheng H, Lok GT, Deng Y, Huen MS, Chen J, Jin J and Chang S: The E3 ubiquitin ligase Rnf8 stabilizes Tpp1 to promote telomere end protection. Nat Struct Mol Biol 18: 1400-1407, 2011

27. Halaby MJ, Hakem A, Li L, El Ghamrasni S, Venkatesan S, Hande PM, Sanchez O and Hakem R: Synergistic interaction of Rnf8 and p53 in the protection against genomic instability and tumorigenesis. PLoS Genet 9: e1003259, 2013.

28. Kaur RP, Vasudeva K, Kumar R and Munshi A: Role of p53 Gene in breast cancer: Focus on mutation spectrum and therapeutic strategies. Curr Pharm Des 24: 3566-3575, 2018.

29. Duffy MJ, Synnott NC and Crown J: Mutant p53 in breast cancer: Potential as a therapeutic target and biomarker. Breast Cancer Res Treat 170: 213-219, 2018.

30. Zhou T, Yi F, Wang Z, Guo Q, Liu J, Bai N, Li X, Dong X, Ren L, Cao L and Song X: The functions of DNA damage factor RNF8 in the pathogenesis and progression of cancer. Int J Biol Sci 15: 909-918, 2019.

31. Wang M, Chen X, Chen H, Zhang X, Li J, Gong H, Shiyan C and Yang F: RNF8 plays an important role in the radioresistance of human nasopharyngeal cancer cells in vitro. Oncol Rep 34: 341-349, 2015.

32. Fu R, Han CF, Ni T, Di L, Liu LJ, Lv WC, Bi YR, Jiang N, He Y, Li HM, et al: A ZEB1/p53 signaling axis in stromal fibroblasts promotes mammary epithelial tumors. Nat Commun 10: 3210 , 2019.

This work is licensed under a Creative Commons Attribution-NonCommercial-NoDerivatives 4.0 International (CC BY-NC-ND 4.0) License. 